\title{
Nanoscale
}

Check for updates

Cite this: Nanoscale, 2020, 12, 24419

\section{Introducing electrical conductivity to metal- organic framework thin films by templated polymerization of methyl propiolate $\uparrow$}

\author{
Beren Sen, ${ }^{a}$ Jaciara C. C. Santos, (D) ${ }^{a}$ Ritesh Haldar, (D) a Qiang Zhang, ${ }^{a}$ \\ Tawheed Hashem, (DD ${ }^{a}$ Peng Qin, ${ }^{a}$ Ying Li, ${ }^{a}$ Frank Kirschhöfer, ${ }^{a}$ \\ Gerald Brenner-Weiss, (D) a Hartmut Gliemann, ${ }^{a}$ Lars Heinke, (D) a \\ Christopher Barner-Kowollik, (D) ${ }^{b, c}$ Alexander Knebel (D) *a and Christof Wöll (DD*a
}

Received 23rd September 2020 Accepted 11th November 2020

DOI: $10.1039 /$ dOnr06848a

rsc.li/nanoscale

\begin{abstract}
We herein present a case study on the templated, Pd-catalyzed polymerization reaction of methyl propiolate in the confined pore space of three different surface anchored metal-organic framework (SURMOF) systems in order to introduce electrical conductivity to MOF thin films and provide predictions for potential device integrations. To gain comprehensive insight into the influence of the template on polymerization, we chose $\mathrm{Cu}(\mathrm{bpdc}), \mathrm{Cu}_{2}(\mathrm{bdc})_{2}(\mathrm{dabco})$ and HKUST-1 because of their different types of pore channels, 1D, quasi-1D and 3D, and their free pore volumes. Well-defined MOF thin films were prepared using layer-by-layer deposition, which allows for the application of several characterization techniques not applicable for conventional powder MOFs. With SEM, AFM, XRD, MALDI-ToF/MS, ToF-SIMS and QCM, we were able to investigate the behaviour of the polymer formation. For lower dimensional pore channels, we find a depot-like release of monomeric units leading to top-layer formation determined by desorption kinetics, whereas for the 3D channels, quick release of an excess amount of monomers was observed and polymerization proceeds perfectly. Despite polymerization issues, control over the maximum chain lengths and the molecular weight distribution was achieved depending on the dimensionality of the pore systems. For the HKUST-1 system, polymerization was optimized and we were able to measure the electrical conductivity introduced by the conjugated polymer inside the channels.
\end{abstract}

\section{Introduction}

In recent years, metal-organic frameworks (MOFs) have been used as templates for polymerization reactions of different kinds of monomers, thus exploiting the MOF pore space to control and steer the polymerization process..$^{1-3}$ We herein present a comparative study not only focusing on polymerization control but also on the introduction of electrical conductivity to MOFs. MOFs, as a class of ordered crystalline porous materials consisting of inorganic metal ions and bridging organic linkers, are versatile materials but mostly insulators. ${ }^{4-7}$

\footnotetext{
${ }^{a}$ Institute of Functional Interfaces, Karlsruhe Institute of Technology,

Hermann-von-Helmholtz-Platz 1, 76344 Eggenstein-Leopoldshafen, Germany. E-mail: alexander.knebel@kit.edu,christof.woell@kit.edu

${ }^{b}$ Centre for Materials Science, School of Chemistry and Physics, Queensland University of Technology (QUT), 2 George Street, QLD 4000Brisbane, Australia ${ }^{c}$ Institute of Nanotechnology, Hermann-von-Helmholtz-Platz 1,

76344 Eggenstein-Leopoldshafen, Germany

$\dagger$ Electronic supplementary information (ESI) available. See DOI: 10.1039/ d0nr06848a
}

The diversity of metal ions and linker molecules provides the possibility to tune pore sizes, intrinsic surface areas, pore windows, pore geometries and also pore surface functionalities. ${ }^{8,9}$ Recently, layered 2D conductive MOFs have been developed as high-performance electrode materials. ${ }^{10-12}$ However, the cavities of MOFs open up highly interesting spaces for different kinds of applications, such as catalysis, ${ }^{13}$ gas storage, ${ }^{14}$ separation $^{15,57}$ and environmental remediation. ${ }^{16}$ Most important for this study is that the pores and pore-channels of MOFs provide confined space for chemical reactions, e.g. polymerization, to occur. ${ }^{13,17-20}$ The formation of polymers inside the pores of MOFs has been reported for methacrylate, ${ }^{21}$ aromatic acetylene, ${ }^{22}$ vinyl monomers, ${ }^{23,24}$ substituted acetylenes, ${ }^{25}$ graphene nanoribbons ${ }^{26}$ and polyacrylonitrile. $^{27}$ In the present study, we focused on the polymerization of methyl propiolate (MP) to the conjugated poly(methyl propiolate) to introduce conductivity to SURMOF (surface anchored MOF) systems ( $c f$. Fig. 1).

First of all, confining polymerization reactions inside the pores of MOFs can lead to several advantages over the conventional bulk polymerization reactions. The key factors involved 
(a)

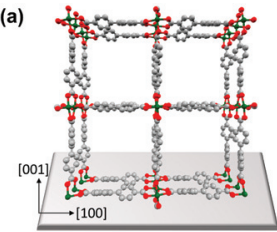

(d)

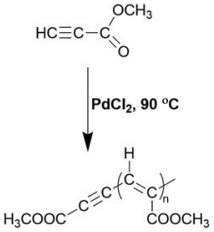

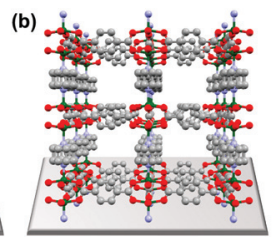

(e)

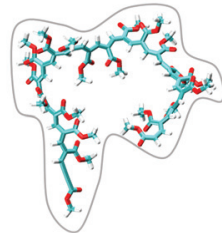

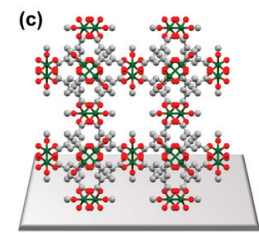

(f)

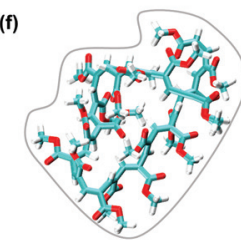

Fig. 1 Visualization of the structure of (a) 1 (SURMOF-2, Cu(bpdc)), (b) $2\left(\mathrm{Cu}_{2}(\mathrm{bdc})_{2}(\mathrm{dabco})\right)$, and (c) 3 (HKUST-1, Cu(btc)). Color scheme: C (gray), $\mathrm{O}$ (red), N (purple), and $\mathrm{Cu}$ (green). (d) MP polymerization catalyzed by $\mathrm{PdCl}_{2}$. (e) Molecular dynamics simulation of the (e) initial structure of poly(methyl propiolate) and the ( $f$ ) final coiled structure of poly (methyl propiolate).

in using MOFs as templates are (i) the controllability of the molecular weight of polymer chains, ${ }^{28,29}$ (ii) the morphology and arrangement of polymers, ${ }^{30-32}$ (iii) the reduction of branching during polymerization ${ }^{23}$ and (iv) the pre-determination of the reaction direction by encapsulation of monomers. ${ }^{28}$

SURMOF thin films have rarely been used for polymerization reactions ${ }^{33-35}$ and are known to have several benefits over the conventional powdered form of MOF materials: for MOF thin films non-conventional characterization techniques can be applied to follow the polymerization reaction, due to the uniformity of the MOF film. ${ }^{34-40}$ Here, we show that SURMOFs can be used as model systems to monitor the controlled polymerization reaction utilizing 3 different pore systems of the studied MOFs.

Pd-catalysed polymerization of methyl propiolate, a substituted acetylene, was performed in 1D and 3D nanochannels using three different SURMOF model systems: $\mathrm{Cu}($ bpdc) (1) $\left(\right.$ bpdc $=4$,4-biphyenyldicarboxylic acid), $\mathrm{Cu}_{2}(\mathrm{bdc})_{2}$ dabco (2) (bdc $=1,4$-benzenedicarboxylate, dabco $=1,4$-diazabicyclo (2.2.2)octane), and HKUST-1 (3) (Hong Kong University of Science and Technology-1). Interestingly, the length of the polymer formed by the polymerization reaction was found to depend on the particular type of MOF used, with a maximum length of $n=22$ in the channels of 1 , whereas in the channels of 2 , the polymer led to a length of up to $n=19$. In the 3D channels of 3 , HKUST- 1 , the polymers were found to have a length of up to $n=23$, the same as that in bulk polymerization. We carried out comprehensive studies identifying the benefits and drawbacks related to the use of $1 \mathrm{D}$ and $3 \mathrm{D}$ pore systems for polymerization reactions. We find depot-effects by loading and unloading experiments with the QCM sensors, which show significantly different desorption kinetics between species from the lattices of MP; with 1 leading to a top-layer formation in the former case, and successful polymer formation in the latter case for 3 .

\section{Experimental}

\section{Chemicals}

Copper acetate monohydrate $(\geq 98 \%)$, palladium chloride (99\%), MUD (11-mercapto-1-undecanol, 97\%), bdc (1,4-benzenedicarboxylate, 98\%), 16-mercaptohexadecanoic acid (MHDA), dabco(1,4-diazabicyclo(2.2.2)octane ( $\geq 99 \%)$ and DCTB (trans-2-[3-(4-tert-butylphenyl)-2-methyl-2-propenylidene] malononitrile, $\geq 99 \%$ ), hydrochloric acid (37\%), glacial acetic acid, tetrahydrofuran (THF, $(\geq 99.9 \%)$ ) and chloroform $(\geq 99.5 \%)$ were purchased from Merck, Germany. Methyl propiolate (99\%) was purchased from Alfa-Aesar, Germany. Absolute ethanol ( $\geq 99.8 \%$ ) was purchased from VWR chemicals. The substrates used for SURMOF synthesis consisted of a $100 \mathrm{~nm} \mathrm{Au} / 5 \mathrm{~nm}$ Ti metal bilayer deposited on p-doped (100) Si wafers and plain p-doted (100) Si wafers. The Au surface was functionalized by a self-assembled monolayer of MUD before SURMOF synthesis. All chemicals were used as received, without further purification.

\section{X-ray diffraction (XRD)}

$\mathrm{X}$-ray diffraction was performed using a D8 ADVANCE Bruker AXS diffractometer with $\mathrm{Cu} K \alpha$ radiation $(\lambda=1.5418 \AA)$ in $\theta-\theta$ geometry with a position sensitive LynxEye detector and a variable divergence slit. The samples were investigated with a scan speed of $1 \mathrm{~s}$ and an increment of $0.02^{\circ}$ per step.

\section{Modelling the SURMOF structures by loading MP and $\mathbf{P d C l}_{2}$}

The software package BIOVIA Material studio 2018 was used to create appropriate models of $\mathbf{M P}$ and $\mathrm{PdCl}_{2}$ loaded 1, 2 and 3 MOF structures. Each unit cell was filled with one MP and $\mathrm{PdCl}_{2}$. The structure models were then used to simulate XRD patterns for comparison with the experimental data. No structural optimization using e.g. force fields was carried out for these model structures.

\section{Simulation of poly(methyl propiolate)}

Force field simulations of the coiling behavior of polyMP with $n=20$ were performed using CgenFF version 4.0 with a CHARMM36 force field being applied..$^{41-44}$ Coiling simulation started with the steepest descent minimization, followed by a Canonical ensemble (NVT) simulation of 100 ps using a V-rescale thermostat, and then by an NVT simulation of $10 \mathrm{~ns}$ using a Nosé-Hoover thermostat. All simulations were performed using GROMACS 2019.2 with periodic boundary conditions being applied.

\section{ToF-SIMS (time-of-flight secondary ion mass spectrometry)}

ToF-SIMS experiments were performed on a TOF.SIMS ${ }^{5}$ instrument (IONTOF GmbH, Münster, Germany). The base pressure is $7 \times 10^{-9}$ mbar. $\mathrm{Bi}_{3}{ }^{+}$ions, $25 \mathrm{keV}, 0.27 \mathrm{pA}$ at $125 \mu$ s cycle time, were used as primary ions; $\mathrm{Ar}_{1200}{ }^{+}$ions, $0.25 \mathrm{keV}, 0.7 \mathrm{nA}$, were used as sputter ions. To remove trace amounts of physisorbed MP and to differentiate them from thicker layers of polyMP, dynamic SIMS spectra were recorded in the non-interlaced mode. Therefore, a $700 \times 100 \mu^{2}$ crater was eroded 
(3 scans followed by $1 \mathrm{~s}$ pause), and a concentric field of view of $500 \times 500 \mu \mathrm{m}^{2}$ was analyzed ( 3 frames) until a total sputter time of $600 \mathrm{~s}$ was achieved.

\section{MALDI-ToF/MS}

MALDI-ToF/MS experiments were carried out using a 4800 MALDI-ToF/ToF mass spectrometer (AppliedBiosystems/MDS SCIEX, Foster City, CA) equipped with a Nd:YAG pulsed laser (355 nm wavelength with $<500$ ps pulses and $200 \mathrm{~Hz}$ repetition rate) and using the 4000Series Explorer (V3.5.3) and DataExplorer (V 4.9) software. Data acquisition was performed in the reflector positive ion mode. The selected acquisition range was 600 to $2000 \mathrm{~m} / \mathrm{z}$. Each mass spectrum showed an average of 1000 laser shots over the entire spot.

Prior to the MALDI-ToF/MS analysis, the SURMOF thin films were dissolved by immersion in a solution of $50 \mu \mathrm{L}$ of acetic acid mixed with $0.4 \mathrm{~mL}$ of ethanol, retaining the polymer chains. To prepare a mixture solution for MALDI-ToF/ MS analysis, $0.5 \mu \mathrm{L}$ of the resulting liquid was mixed with $0.5 \mu \mathrm{L}$ of matrix solution consisting of $10 \mathrm{mg}$ of DCTB in $1 \mathrm{~mL}$ of THF. After digestion of the SURMOF, $5 \mu \mathrm{L}$ of the resulting solution was spotted and air-dried on the MALDI target.

\section{SEM and EDXM}

A TESCAN Vega 3 with a tungsten filament electron source was used for scanning electron microscopy (SEM) analysis. Images were recorded using a working distance of 6-14 $\mathrm{mm}$, with an emission voltage of 8-10 kV. For energy dispersive X-ray spectroscopy (EDXS) and mapping (EDXM), a Bruker XFlash Detector $610 \mathrm{M}$ was used, always with an emission voltage of $10 \mathrm{kV}$. To prevent artifacts caused by electrical charging of the SURMOFs, the samples were sputtered with a Bal-Tec MCS 010 coating system using an $\mathrm{Au}-\mathrm{Pd}$ target. Regarding the choice of the target, we found that the determination of catalytic Pd amounts in the MOF sample is not possible with sufficient accuracy and the underlying Au-layer in HKUST-1 is not necessary to quantify, and thus the $\mathrm{Au}-\mathrm{Pd}$ target was used for the best possible resolution.

\section{AFM}

The AFM micrographs were recorded at room temperature using a Bruker Dimension Icon Atomic Force Microscope in alternating current mode (AC mode) in air using MikroMasch NSC15 probes, with a scan rate of $0.6 \mathrm{~Hz}$ and 400 scan lines. A minimum number of modifications are performed in the raw data. Pristine and polymerized samples were sonicated in $1 \mathrm{mM}$ diluted hydrochloric acid solution for 30 minutes in order to remove the SURMOFs. Then, the solution containing the polymer was dropcast onto a smooth Si substrate and investigated by AFM. ${ }^{45}$

\section{Electrical conductivity measurements}

The electrical conductivity of the sample was investigated by 2-probe current-voltage measurements using a Keithley 2635B Source Meter. The sample was grown on interdigitated gold electrodes on glass substrates from Metrohm. The gap width between the electrodes increased to $5 \mu \mathrm{m}$, with a total gap length of $3.38 \mathrm{~m}$. Electrical characterization was carried out under a pure nitrogen atmosphere at room temperature (298 K). Before the experiment, the sample was equilibrated under the nitrogen atmosphere for more than one hour, in order to allow all volatile guest molecules to desorb from the pores. As a reference to the polymer-filled HKUST-1 sample, an empty HKUST-1 sample was used. For more details of the experimental setup used for conductivity measurements, see the study by Garg et al. ${ }^{46}$

\section{Synthesis of SURMOF templates}

In this study, thin films of $\mathrm{Cu}(\mathrm{bpdc})(\mathbf{1}), \mathrm{Cu}_{2}(\mathrm{bdc})_{2}$ (dabco) (2) and HKUST-1 (3) were selected as templates for methyl propiolate polymerization. Thin films of $\mathrm{Cu}(\mathrm{bpdc})(\mathbf{1})$ and HKUST-1 (3) were grown using layer-by-layer deposition via the spray coating method. ${ }^{33} \mathrm{Si}$ substrates were functionalized by UV treatment for 30 minutes prior to synthesis, whereas the $\mathrm{Au}$ substrates were functionalized using MHDA (16-mercaptohexadecanoic acid) solution. For MHDA functionalization, gold substrates were immersed in $1 \mathrm{mM}$ ethanolic MHDA solution for $72 \mathrm{~h}$. The activated substrates for $\mathbf{1}$ were mounted on the sample holder and subsequently sprayed with a $1 \mathrm{mM}$ ethanolic solution of $\mathrm{Cu}\left(\mathrm{CH}_{3} \mathrm{COO}\right)_{2} \cdot \mathrm{H}_{2} \mathrm{O}$ and a $0.2 \mathrm{mM}$ ethanolic solution of 4,4-biphyenyldicarboxylic acid for 30 spray cycles. The thin films of $\mathbf{3}$ were also prepared using the same method. The metal solution was the same as that of $\mathbf{1}$, whereas the linker solution was $0.2 \mathrm{mM}$ 1,3,5-benzenetricarboxylic acid in ethanol.

Thin films of $\mathrm{Cu}_{2}(\mathrm{bdc})_{2}$ (dabco) (2) were grown on $\mathrm{Au}$ and $\mathrm{Si}$ substrates using layer-by-layer deposition via a pump system. ${ }^{47}$ Prior to SURMOF synthesis, the gold substrates were functionalized by SAMs deposited from a MUD (11-mercapto-1-undecanol) solution. ${ }^{48}$ For MUD functionalization, the gold substrates were immersed in $1 \mathrm{mM}$ ethanolic MUD solution for $24 \mathrm{~h}$. After functionalization, the $\mathrm{Au}$ substrates were rinsed with pure ethanol and dried under a $\mathrm{N}_{2}$ stream. $1 \mathrm{mM}$ $\mathrm{Cu}\left(\mathrm{CO}_{2} \mathrm{CH}_{3}\right)_{2} \cdot \mathrm{H}_{2} \mathrm{O}$ as the metal solution and a mixture of $0.1 \mathrm{mM}$ bdc(1,4-benzenedicarboxylate) and dabco(1,4-diazabicyclo (2.2.2)octane) as the linker solution were used in order to grow 2. After functionalization of the substrates, they were placed inside the sample holder of a pump system. ${ }^{49}$ During synthesis, the substrates were immersed in the ethanolic copper acetate solution for 10 minutes, whereas the immersion time in the linker solution was 20 minutes. 40 deposition cycles were run at $60{ }^{\circ} \mathrm{C}$.

\section{Quartz crystal microbalance}

A Q-Sense E4 Auto4 device was used with a Q-Sense QCM sensor, diameter $14 \mathrm{~mm}$, thickness $0.3 \mathrm{~mm}$, AT cut, and fundamental frequency 4.95 MHz. SURMOF systems 1 (deposited on the Au coated QCM sensor) and 3 (deposited on the $\mathrm{SiO}_{2}$ coated QCM sensor) were synthesized using the same procedure as that described above for 40 cycles. Temperatures of $25{ }^{\circ} \mathrm{C}$ and $40{ }^{\circ} \mathrm{C}$ were used for adsorption and desorption experiments. Flowrates for $\mathrm{Ar}$ and $\mathrm{N}_{2}$ were always kept at 
$100 \mathrm{ml} \mathrm{min}^{-1}$ using a mass flow controller. The samples were activated under Ar-flow at $40{ }^{\circ} \mathrm{C}$ overnight, cooled down to $25{ }^{\circ} \mathrm{C}$ and measured under $\mathrm{N}_{2}$-flow. For dosing of MP, $\mathrm{N}_{2}$ was bubbled through pure MP until a steady state is reached, further followed by desorption with pure $\mathrm{N}_{2}$. For complete desorption from the frameworks, heating to $40{ }^{\circ} \mathrm{C}$ was necessary.

\section{Methyl propiolate loading and polymerization in SURMOFs}

Before the loading of methyl propiolate (MP), SURMOFs were activated under vacuum at $80^{\circ} \mathrm{C}$ for 6 hours in order to completely remove the guest solvents. For the loading step, $400 \mu \mathrm{L}$ of methyl propiolate was mixed with a trace amount of palladium chloride $\left(\mathrm{PdCl}_{2}\right)$ catalyst. ${ }^{50}$ This solution was dropcast onto the activated SURMOFs and left undisturbed for 2 hours in a glass vial at room temperature. To remove the residual monomer, the samples were washed with chloroform prior to polymerization. Afterwards, the MP loaded SURMOFs (MP@1, 2 , 3) were placed in an oven at $90{ }^{\circ} \mathrm{C}$ for 5 hours for the polymerization of the MP in the SURMOFs (polyMP@1, 2, 3). Finally, the samples were washed with chloroform to remove the unreacted monomer and the excess polymer from the surface for further characterization.

Bulk polymerization of MP was performed with a slight difference to that reported in the literature. ${ }^{50}$ In order to compare the chain length of polyMP in bulk to that synthesized in the pores of the SURMOFs, bulk polyMP was prepared by mixing $400 \mu \mathrm{L}$ of $\mathbf{M P}$ with a trace amount of $\mathrm{PdCl}_{2}$. The mixture was placed in an oven at $90^{\circ} \mathrm{C}$ for 5 hours.

\section{Results and discussion}

Three different SURMOF systems were investigated as host materials for methyl propiolate (MP) polymerization, $\mathrm{Cu}(\mathrm{bpdc})$, also called SURMOF-2 (1), $\mathrm{Cu}_{2}(\mathrm{bdc})_{2}$ (dabco) (2), and HKUST-1 (3). Fig. 1a, b, c shows the structures of 1, 2 and 3, respectively, along with the reaction mechanism for coupling the MP units to yield poly(methyl propiolate) (polyMP) in the presence of $\mathrm{PdCl}_{2}$. The structure of the polymer is shown in Fig. 1e and f. Systems 1 and 2 were deposited on Si wafers using the layer-by-layer synthesis procedure; in the case of $\mathbf{3}$, gold-coated Si-wafers were used. The layer-by-layer procedure is well known to yield very uniform and controllable MOFs with a well-defined orientation on the substrate. SURMOF system 1, which is a member of the SURMOF-2 isoreticular series, ${ }^{33}$ exhibits a sheet like structure, expressing $1 \mathrm{D}$ pore channels that are aligned parallel to the supporting Si-wafer (Fig. 1). The interlayer distance between the planes in structure $\mathbf{1}$ amounts to $5.6 \AA^{51} 2$ has a $3 \mathrm{D}$ pore system, with large square shaped channels of $7.5 \times 7.5 \AA$ perpendicular to the supporting layer. However, in 2, the cubic pore windows oriented parallel to the support have rather small openings with a size of only $3.8 \times$ $4.7 \AA$ (ref. 52) (cf. Fig. 1b). We believe that these openings are too small to allow for diffusion of MP (kinetic diameter $6.2 \AA$ ), and therefore, we consider it as a quasi-2D framework for the polymerization of MP. SURMOF system 3 is an already well- studied and known system, perfect for this comparative study, with a $3 \mathrm{D}$ pore-system exhibiting pore windows with diameters of $9 \AA$ and $6 \AA$ and 3 different cavities with diameters of $14 \AA$, $11 \AA$ and $5 \AA^{53}$ The smallest cavity in 3 is not considered relevant for the polymerization reaction due to the size of MP. Nevertheless, there is sufficient space in HKUST-1 to allow for the formation of continuous polyMP. The polymerization of MP was catalysed by using small amounts of $\mathrm{PdCl}_{2}$. Trace amounts of $\mathrm{PdCl}_{2}$ were first added to pure $\mathbf{M P}$, which was then loaded subsequently into the SURMOF. What all of the MOF systems investigated in this study have in common is that the linkers are connected via Cu-paddle wheel type secondary building units.

Estimates of the sizes of polymer coils formed from polyMP were obtained on the basis of molecular dynamic simulations (Fig. 1e and f), using a long polymer of 20 monomer units.

(a)

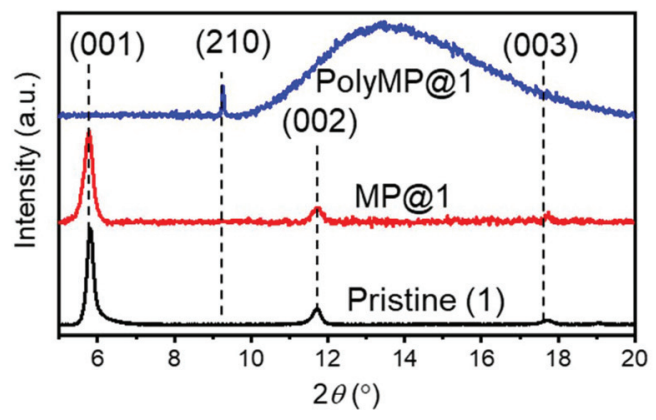

(b)

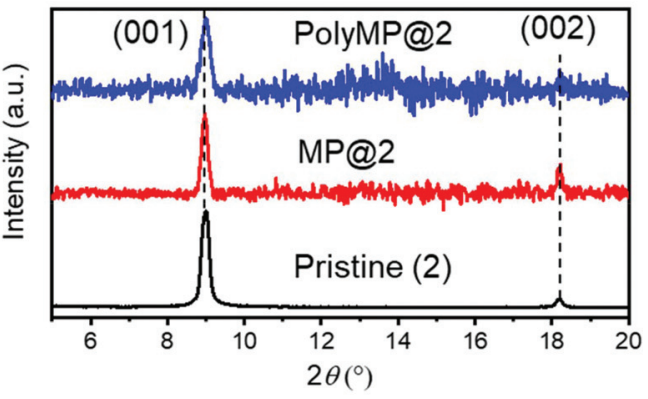

(c)

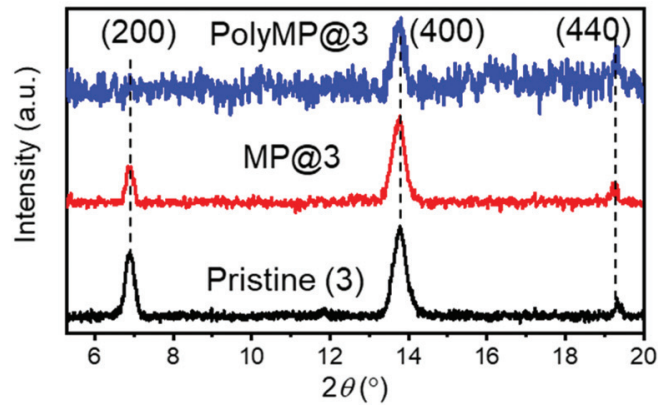

Fig. 2 Out-of-plane XRD patterns of (a) 1, (b) 2 and (c) 3 in the pristine state (black), after MP loading (red) and after polymerization (blue) with normalized intensities. Changes in the form factors upon loading are visible, as well as intensity differences. The detailed modelling of the structure upon monomer loading has been performed and can be found in the ESI. $\dagger$ 
The simulations indicate the formation of coils, as expected, with an average radius of gyration that equals $0.66 \mathrm{~nm}$. The analysis of the coil structure showed that the last frame yielded dimensions of $1.5 \times 1.9 \times 1.8 \mathrm{~nm}$. On the basis of these simulations, we expect that the 22 coils formed should fit into the pores of all three MOF systems.

Upon loading and polymerization, XRD patterns were recorded as shown in Fig. 2. They reveal that MP loading has only a weak influence on the reflexes. Nonetheless, small form factor variations as well as changes in the intensity ratios are observable. This experimental observation is consistent with the simulations obtained for MOF models where the pore contained $1 \mathrm{MP}$ or $\mathrm{PdCl}_{2}$ molecule, leading to comparable results: the modelled MP loading provides an estimation of the experimental polyMP XRD data. The modelled patterns of MP and $\mathrm{PdCl}_{2}$ loading are shown separately for $\mathbf{1}, 2$ and 3 and the changes are observed in the XRD patterns as shown in Fig. S1.† From the simulated MP@1, MP@2 and MP@3 XRD patterns (Fig. S1 $\dagger$ ), it can be observed that the crystallographic changes are consistent with the experiments ( $c f$. Fig. 2). For the SURMOF system 1, the (001) and (002) peaks disappeared after polymerization, while a new (210) peak emerged. According to modelling, after loading of $\mathbf{M P}$ and $\mathrm{PdCl}_{2}$ into 1, the (210) reflex appears (Fig. S1 $\dagger$ ). Comparing the experimental and simulated data of the samples, we conclude that the appearance of the reflex for the lattice plane (210) is attributed to the successful polymerization (Fig. S1a†). For 2, the loading with MP and the subsequent polymerization to polyMP@2 showed changes in the reflex intensities, together with introduced crystal strain, and visible changes of the form factors (Fig. 2b).
The XRD pattern of 3 showed that the (200) peak disappeared, whereas the (400) peak was still observable after polymerization. After MP loading, the form factors changed as well (Fig. 2c). In modelling the structure of $\mathbf{3}$, the effects found experimentally are stronger, yet a comparable change is observed. After the loading of MP into SURMOF system 3, the intensity of the (200) peak is decreased (Fig. S1c $\dagger$ ). From all XRD patterns, it can be concluded that MP loading and polymerization have a strong effect on the reflex intensities and form factors, but the MOF structure remains crystalline.

\section{Morphological studies of the SURMOFs upon polymerization}

The cross-sections of pristine and polymerized samples of $\mathbf{1}, 2$ and 3 were characterized by scanning electron microscopy (SEM) and energy dispersive X-ray spectroscopy and mapping (EDXS and EDXM) in order to assess if visible changes appear after polymerization ( $c f$. Fig. 3). Further characterization using mass spectroscopy was important to investigate the products of the polymerization reaction, which is given in Fig. 5 .

The SEM pictures and EDXM in Fig. 3 show that the pristine SURMOF layers are all close to $200-300 \mathrm{~nm}$ in thickness. After the polymerization reaction, a number of changes can be observed. It can be clearly seen from SEM analysis together with EDXM that a thick layer of polymer covers the surface of $\mathbf{1}$ (Fig. 3a and b) after the polymerization reaction. We subsequently evidence that monomers slowly desorbing from the lattice of 1 yield a surface covering polymer film, the SURMOF thin film. This diffusion out of the SURMOFs could result from the fact that the pore channels of $\mathbf{1}$ are aligned parallel to the underlying Si-wafer, making the desorption kinetics slower than that of e.g. 3 (Fig. $3 \mathrm{c}$ and $\mathrm{d}$ ) and resulting in a depot-like (a)

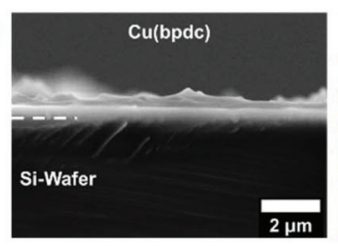

(c)

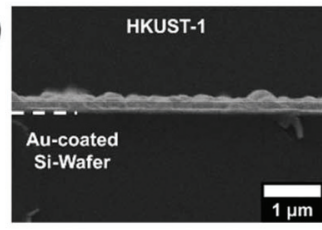

(e)

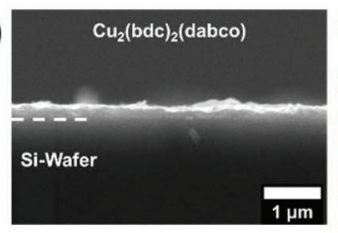

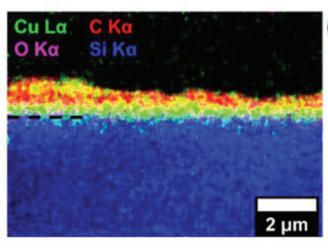
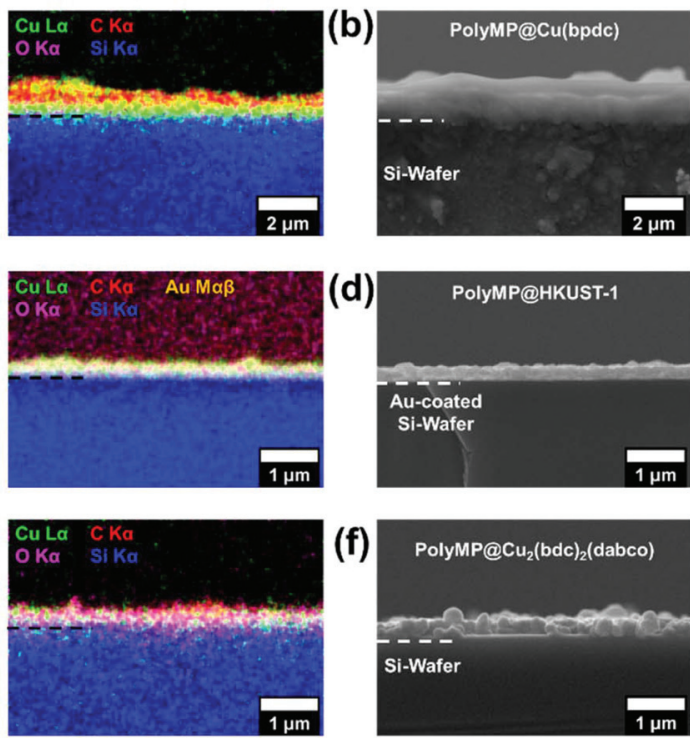

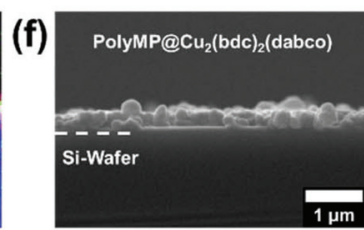

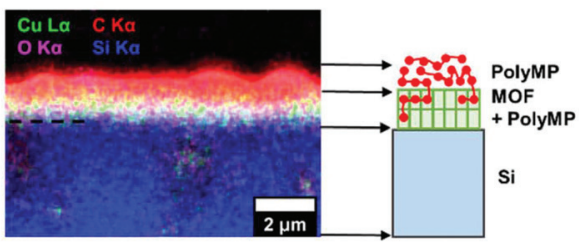
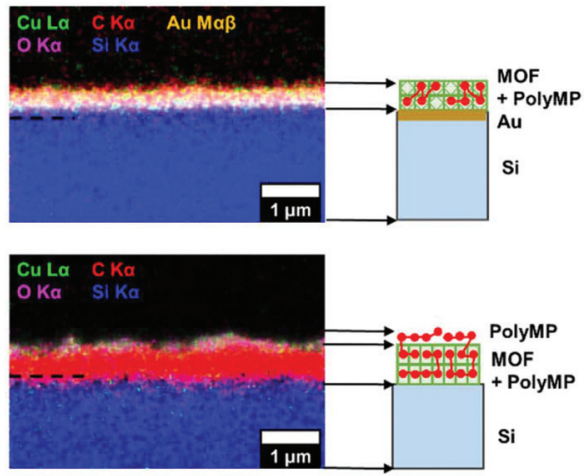

Fig. 3 SEM images and the corresponding EDXM of (a) pristine 1, (b) polyMPa1, (c) pristine 3, (d) polyMPa3, (e) pristine 2, and (f) polyMPa2. For 1 and polyMP@1, the formation of a clear, strong top-layer is visible, whereas for 3 and polyMP@3, no top-layer formation is observed. Note that due to the strong intensity of carbon from (e) to $(f)$, the MOF signal almost diminishes, but from the thickness difference, we assume the formation of a small top-layer. The cartoons on the right side of (b), (d) and ( $f$ ) show the observations of polyMP formation. 
effect. The EDX spectra of the SURMOF films (Fig. S2-S4†) show an increase in the C-signal to Cu-signal ratio, which is evidence for polymerization inside the MOFs in all cases, and an overall effect after polymerization was expected for all samples. With the distribution of the carbon signal in the EDXM, we can see where the polymer is formed, which is definitely a surface covering top-layer for $\mathbf{1}$ and $\mathbf{2}$, and is not observed for 3 (Fig. 3).

Investigations show that the thickness of the polymer toplayer of 1 has a linear dependency on the thickness of the SURMOFs (Fig. S5 $\dagger$ ), which is also attributed to the slow and steady desorptive release of $\mathbf{M P}$ from the framework of $\mathbf{1}$, as can also be shown by quartz crystal microbalance (QCM) experiments (Fig. 4c and Fig. S6†). For 3, this phenomenon of a polymer top-layer is not found. Loading experiments of MP in SURMOFs $\mathbf{1}$ and $\mathbf{3}$ were performed in order to determine the adsorption/desorption kinetics of the thin films (Table S1 $\dagger$ ), where the difference is observable in the normalized mass change over the time diagram in Fig. 4c. The desorption kinetics of MP from 3 is much faster than that of MP from 1 (Fig. 4, Fig. S6 and Table S1 $\dagger$ ). Thus, the desorption

(a)

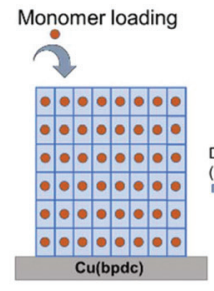

(b)

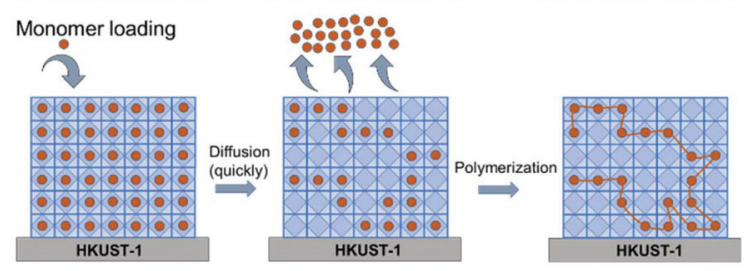

(c)

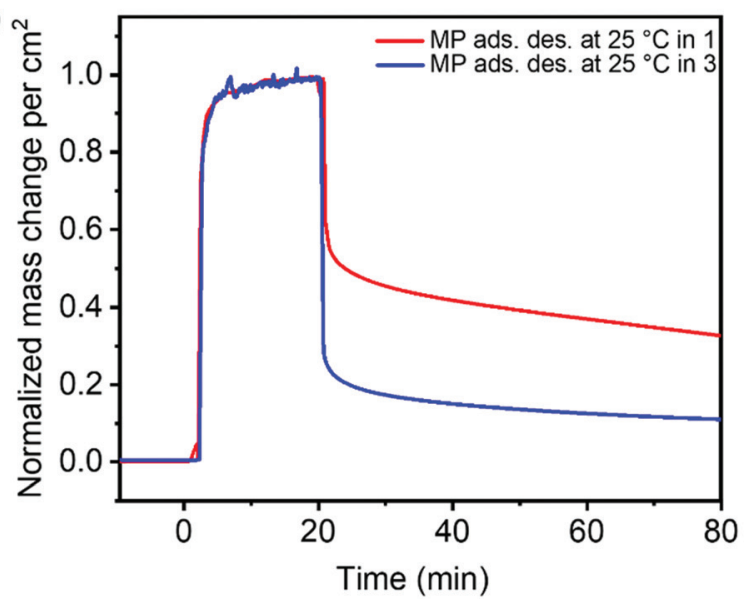

Fig. 4 Schematic diagram showing the depot effect of (a) MP diffusion from 1 and (b) quick release from the framework of 3, followed by polymerization. In (c), the normalized mass change from the adsorption and desorption of MP on 1 and 3 measured using a QCM is shown. kinetics of 1 can be determined from the release of the monomers from a depot, which gives coherent results regarding the top-layer formation observed in SEM images ( $c f$. Fig. 3b and 4a). The depot-effect occurs due to very slow time constants of $T_{0.5} \approx 2 \mathrm{~min}$ and $T_{0.8} \approx 144 \mathrm{~min}$ for 1 , so a top layer can be formed through long term release of MP, whereas MP molecules were desorbed within $T_{0.8} \approx 2.6$ min in 3 during bursttype release, which makes top-layer formation impossible ( $c f$. Table S1† and Fig. 3d). The XRD patterns and SEM cross-sections of the SURMOFs on the QCM sensors are shown in Fig. S7. $\dagger$

For 2, we observe that a thinner layer of the polymer covers the surface (Fig. 3e and f). We assume that SURMOF system 2, due to its quasi-1D pore network (and because of the kinetic diameter of MP), has a release kinetics in-between the poresystems 1 and 3. Thus, desorption kinetics also triggers toplayer formation. Nevertheless, it seems less limited than for $\mathbf{1}$ from SEM analysis. In EDXM, no differentiation between the polymer and the MOF is observable for 2 (Fig. 3f), leading to the assumption that the polymer is additionally equally well distributed inside the pores of 2 as is the case for 3 .

However, for 3 as the desired result, not even a thin toplayer is observable from the SEM images or EDXM and thus no covering of the surface after polymerization ( $c f$. Fig. $3 c$ and d). From EDXM we observe that, for pristine 3, the carbon signal is very low, so that the C-signal was increased for visualization (resulting in the C-signal artefacts in the background) (Fig. 3c). In Fig. 3d, the C-signal has the same strength as that of the $\mathrm{Cu}$-signal, with no observable top-layer, which is already an indicator for successful polymerization inside the pores of 3 . However, all quantitative EDX spectra can be found in the ESI 2-4. $\dagger$

The polymer morphology was further observed using AFM, which is shown in Fig. 5. Fig. 5a and b show the polymer from bulk synthesis, deposited on a flat Si substrate, whereas Fig. 5c and $\mathrm{d}$ show the polymer obtained from SURMOF system 3 after chemical decomposition followed by a similar deposition procedure on a Si substrate. It can be concluded that the bulk polymer forms large, spherical, agglomerated particles. The

(a)

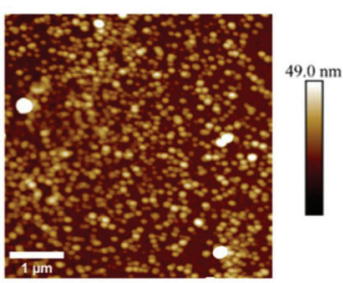

(b)

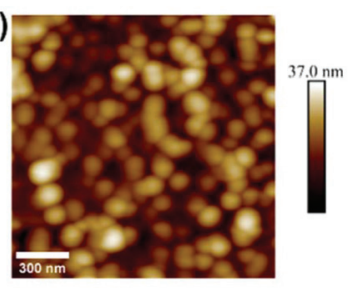

(c)

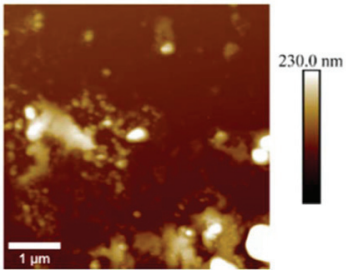

(d)

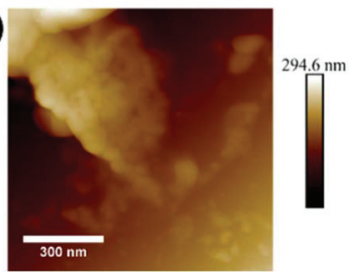

Fig. 5 AFM images of the (a, b) bulk polymer and (c, d) polymer from the SURMOF deposited on a smooth surface. 
polymer formed in the SURMOF looks completely different, and it appears more like a polymer film containing fibers and very tiny, agglomerated particles.

\section{Molecular weight distribution of polyMP formed in SURMOFs \& depth profile analysis of the monomer loaded and polymerized samples}

MALDI-ToF/MS was used in order to determine the chain lengths and molecular weight distribution of the polymers formed in 1, 2 and 3. Therefore, polyMP@1, polyMP@2 and polyMP@3 were immersed in a mixture of ethanol and acetic acid solution in order to dissolve the SURMOF systems. The solution containing polyMP from the samples was analyzed by MALDI-ToF/MS. The molecular mass of a monomeric MP unit is $84.07 \mathrm{~g} \mathrm{~mol}^{-1}$, which is clearly observable in the size distribution of the polymer in the mass spectrum. The masses shown in the spectra (Fig. 6a-c) are sodium adduct masses. Chains of polyMP are observed in $\mathbf{1}$ with a maximum length of up to $n=22$ (Fig. 6a), in 2 with a length of up to $n=19$ (Fig. 6b) and in 3 with a length of up to $n=23$ (Fig. 6c). The full isotopic pattern overlays are shown in Fig. $\mathrm{S} 8, \dagger$ while the isotope patterns of peak $n=8$ for the 3 systems are shown in Fig. S9. $\dagger$ For the bulk polymer formed by the reaction in solution, which we used as a comparative sample, a maximum chain length of up to $n=23$ for this short-chain polymer is observable (Fig. S10†). With this in mind, in the context of SEM analysis (Fig. 3) and polymer coil MD simulation (Fig. 1f), we can conclude that the pore-volume has a strong influence, especially in 1D pore-channels, on the chain length and thus, on the molecular weight distribution. If the polymer is formed mainly on the top-layer (as for polyMP@1), we clearly expect the molecular weight distribution to equalize the molecular weight distribution of the bulk. Surprisingly, we still get a comprehensive result for polyMP@1, with $n=22$, underlining the thesis of confined polymerization. The polymerization of polyMP@2 in the 1D channels of 2 was much more successful, yielding only $n=19$. Interestingly, polyMP@3 (Fig. 6c) gives the same weight distribution as that of the bulk polymer, but without top-layer formation.

The results obtained in a previous study on polymerization in SURMOF-2 (here system 1) only yielded oligomers. Here, we are able to increase the chain length up to the bulk polymer chain length of $n=19-23$. The resulting polymer in the previous study was rather short with a maximum of $n=4$ for terthiophene and $n=9$ for EDOT $^{34}$ suggesting that using in-plane 1D frameworks, a polymerization reaction is always highly restricted by the diffusion of the species inside the pore system, e.g. in this case yields depot-like release and a subsequent confined polymer and bulk polymer mixture. When going to higher dimensional pore systems, the polymerization reaction is becoming more comparable to bulk polymerization.

To further investigate the influence of the SURMOF template for the polymerization reaction, depth integrated spectral analysis and depth profile analysis were done by ToF-SIMS on all samples in order to validate the formation of the polymer inside the pores of SURMOFs. Therefore, mass spectra of the monomer loaded and polymerized samples are shown in Fig. 5. The depth integrated spectra are shown for the $\mathrm{C}_{4} \mathrm{H}_{3} \mathrm{O}_{2}{ }^{-}$ monomeric units from 1 (Fig. 6d), 2 (Fig. 6e) and 3 (Fig. 6f). In the ToF-SIMS analysis of the monomer loaded samples for $\mathbf{1}, \mathbf{2}$ (a)

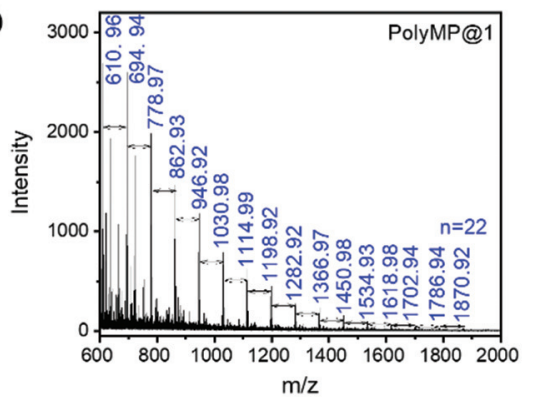

(d)

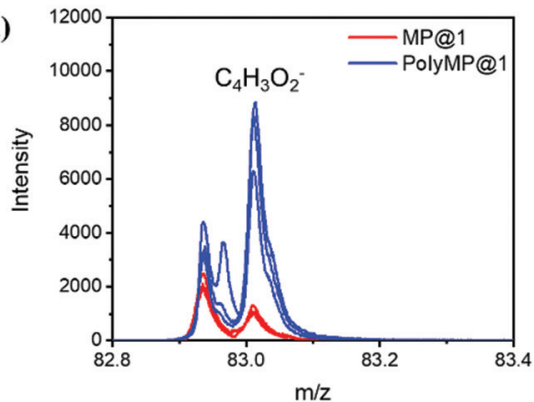

(b)

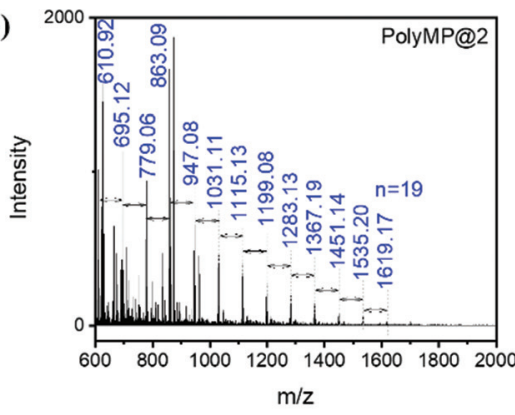

(e)

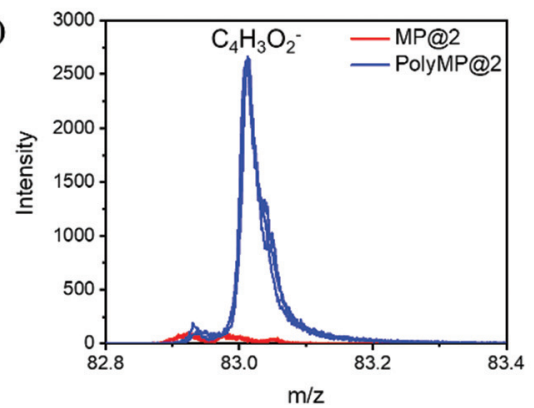

(c)

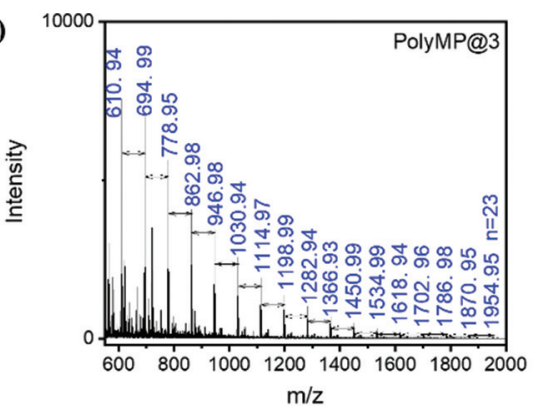

(f)

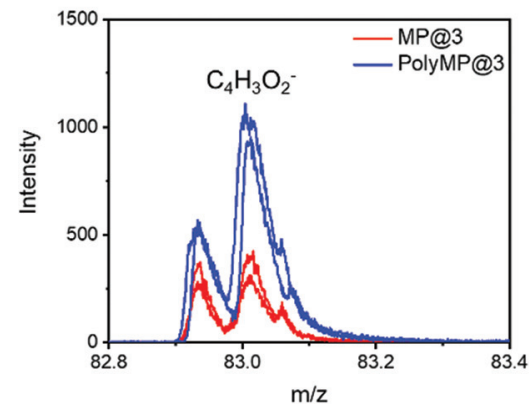

Fig. 6 (a) MALDI-ToF spectrum of (a) polyMPa1, (b) polyMPa2, and (c) polyMPa3 after dissolution in acetic acid/ethanol, depth integrated ToF-SIMS data showing the main fragment of the polyMP repeating unit of (d) MP@1 (red) and polyMP@1 (blue), (e) MP@2 (red) and polyMP@2, and (f) MP@3 (red) and polyMP@3, three different analysed spots on each sample. 
and 3, only rather small amounts of $\mathrm{C}_{4} \mathrm{H}_{3} \mathrm{O}_{2}{ }^{-}$were found, since under UHV conditions the monomer is easily desorbing from the lattice. However, due to a small amount of "sticky" monomer molecules that do not easily desorb from the SURMOF lattices ( $c f$. Fig. S6†), some residual monomers could be detected. Furthermore, it was observed in the depth integrated images that ${ }^{63} \mathrm{Cu}^{-},{ }^{65} \mathrm{Cu}^{-}$and $\mathrm{C}_{4} \mathrm{H}_{3} \mathrm{O}_{2}{ }^{-}$are laterally correlated for the polymerized samples of 1 (Fig. S11 ) $^{2}$ (Fig. S12 $\dagger$ ) and 3 (Fig. S13 ${ }^{\dagger}$ ), respectively. From the ToF-SIMS depth analysis, we can conclude that in our case, even if a toplayer is formed, the polymerization takes place inside the pores for all cases. The depth profiles of the polymerized samples are shown in Fig. S14. $\dagger$ From the ToF-SIMS depth profiles (Fig. S14 $\dagger$ ) normalized on the Cu-signal coming from the MOF, it can be concluded that there is a gradient starting at the top of each SURMOF layer with the highest monomer content, which fades out towards the underlying substrate ( $\mathrm{Si}$ or Au signal).

\section{Electrical conductivity of HKUST-1 after polymerization}

The goal of the present study is the formation of a polymer film completely embedded in a SURMOF. The best result obtained in conductivity measurements ( $c f$. Fig. 7) through polyMP@SURMOF systems could only be achieved for polyMP@3, whereas for the other systems the formation of a thick layer covering the substrate was observed, making them unsuitable for reliable measurement. To further characterize the successful case, the lateral conductivity of $\mathbf{3}$ and polyMP@3 was measured using interdigitated Au-electrodes. The current-voltage curves of the sample are shown in Fig. 7. The FTIR spectra of 3, MP@3 and polyMP@3 used in this measurement are shown in Fig. S15. $\dagger$ The current is proportional to the voltage indicating ohmic conduction behavior. While the polymer-loaded sample shows a current of almost

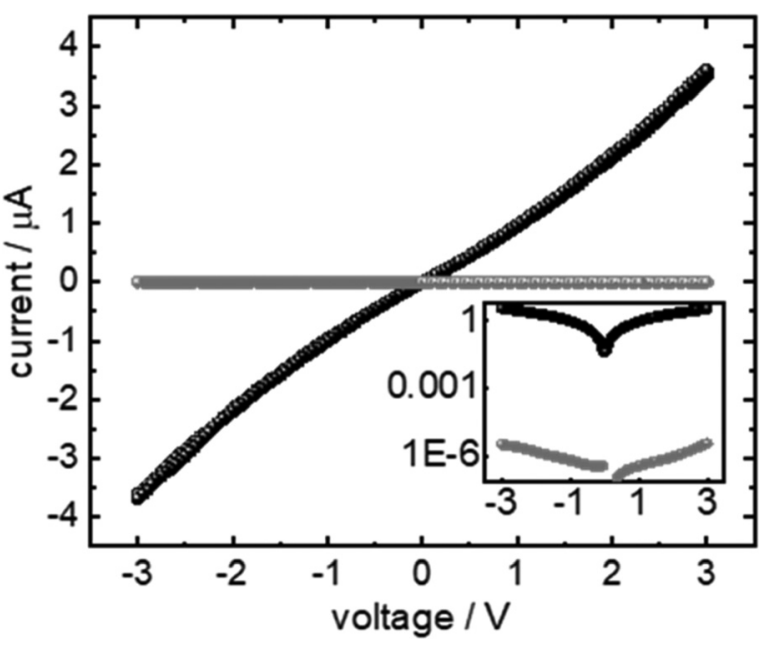

Fig. 7 Conductivity of polyMP@3. The current-voltage curve of the polyMPa3 sample is shown in black, and the current-voltage curve of 3 is shown as a reference in grey. The inset shows the data on a logarithmic scale.
$3.6 \mu \mathrm{A}$ at $3 \mathrm{~V}$, the empty sample shows only a current of $3.3 \mathrm{pA}$. A sample thickness of $200 \mathrm{~nm}$ corresponds to a conductivity of $9 \mu \mathrm{S} \mathrm{m}^{-1}$. This is about 6 orders of magnitude larger than the conductivity of the empty SURMOF system 3, where we obtained a value of $8 \mathrm{pS} \mathrm{m} \mathrm{m}^{-1}$, which is in line with other published values for SURMOFs and for empty HKUST-1 and loaded with 7,7,8,8-tetracyanoquinododimethane. ${ }^{54-56}$

\section{Conclusions}

We have successfully carried out Pd-catalysed polymerization of methyl propiolate in the pores of three different SURMOFs: SURMOF-2, $\mathrm{Cu}_{2}(\text { bdc })_{2}$ (dabco) and HKUST-1. We investigated the influence of pore volumes and pore channels on polymerization, yielding interesting results: the maximum chain length of bulk polymers determined from MALDI-ToF was $n=$ 23, which we obtained for the 3D pore system of HKUST-1, whereas we obtained $n=22$ and $n=19$ for SURMOF-2 and $\mathrm{Cu}_{2}$ (bdc) ${ }_{2}$ (dabco), respectively. In SEM and EDXM analyses, we observed surface-covering top-layer formation for systems SURMOF-2 and $\mathrm{Cu}_{2}(\mathrm{bdc})_{2}$ (dabco), whereas a perfect polymerization reaction is observed for HKUST-1. We investigated the desorption kinetics by QCM experiments and observed quick desorption from HKUST-1, whereas depot-like slow and steady desorption was observed for SURMOF-2, which we assume to be the cause for the top-layer formation in the latter case. From AFM analysis, comparing the bulk polymer to the polymer formed inside HKUST-1, we observe clear control over polymerization: the polymer in the MOF pores forms fibers and tiny particle agglomerates, whereas bulk polymerization yields large spherical particles. Using SIMS analysis we find that, despite the top-layer formation, in all cases polymerization occurs inside the pore systems, which was also highlighted by depth profiling and depth integrated spectra. Finally, for the best working 3D system of HKUST-1, we measured the increase of electrical conductivity after polymerization, which appeared to be 6 orders of magnitude higher than that of the empty HKUST-1. An increase in the electrical conductivity of the MOF system plays a crucial and important role in the potential electrical applications of MOFs, e.g. in sensors or microelectronics, where the insulating, porous structure of the MOF and an electronically conductive electrolyte are needed, such as in supercapacitors. In summary, we introduced lots of novel characterization methods and interpretations of the same, leading towards the prediction of polymerization reactions inside the frameworks of SURMOFs that could further help in developing devices. Introducing electrical conductivity to SURMOFs is of key importance as it leads to various possible applications, such as pseudocapacitors, sensors and actuators.

\section{Conflicts of interest}

There are no conflicts to declare. 


\section{Acknowledgements}

B. S., C. B.-K. and C. W. are thankful for funding through the German Science Foundation (DFG) within the special research field of the SFB 1176 on project C4. A. K. and C. W. are thankful for funding through the German Science Foundation (DFG) within the special research field of the SFB 1176 on project C6. We thank Dr Alexander Welle for the SIMS measurements and acknowledge that the SIMS studies were performed at the Karlsruhe Nano Micro Facility (KNMF). C.B.-K. acknowledges the Australian Research Council (ARC) for funding in the context of a Laureate Fellowship.

\section{Notes and references}

1 B. V. K. J. Schmidt, Macromol. Rapid Commun., 2020, 41, 1900333.

2 U. S. F. Arrozi, V. Bon, C. Kutzscher, I. Senkovska and S. Kaskel, Dalton Trans., 2019, 48, 3415-3421.

3 M. Rivera-Torrente, P. D. Pletcher, M. K. Jongkind, N. Nikolopoulos and B. M. Weckhuysen, ACS Catal., 2019, 9, 3059-3069.

4 S. Kitagawa, R. Kitaura and S. Noro, Angew. Chem., Int. Ed., 2004, 43, 2334-2375.

5 O. M. Yaghi, M. O’Keeffe, N. W. Ockwig, H. K. Chae, M. Eddaoudi and J. Kim, Nature, 2003, 423, 705-714.

6 S. L. James, Chem. Soc. Rev., 2003, 32, 276-288.

7 J. R. Long and O. M. Yaghi, Chem. Soc. Rev., 2009, 38, 12131214.

8 S. M. Cohen, Chem. Rev., 2012, 112, 970-1000.

9 H. Deng, S. Grunder, K. E. Cordova, C. Valente, H. Furukawa, M. Hmadeh, F. Gándara, A. C. Whalley, Z. Liu, S. Asahina, H. Kazumori, M. O'Keeffe, O. Terasaki, J. F. Stoddart and O. M. Yaghi, Science, 2012, 336, 1018-1023.

10 G. Skorupskii, B. A. Trump, T. W. Kasel, C. M. Brown, C. H. Hendon and M. Dincă, Nat. Chem., 2020, 12, 131136.

11 R. Dong, Z. Zhang, D. C. Tranca, S. Zhou, M. Wang, P. Adler, Z. Liao, F. Liu, Y. Sun, W. Shi, Z. Zhang, E. Zschech, S. C. B. Mannsfeld, C. Felser and X. Feng, Nat. Commun., 2018, 9, 2637.

12 D. Sheberla, L. Sun, M. A. Blood-Forsythe, S. Er, C. R. Wade, C. K. Brozek, A. Aspuru-Guzik and M. Dincă, J. Am. Chem. Soc., 2014, 136, 8859-8862.

13 J. Lee, O. K. Farha, J. Roberts, K. A. Scheidt, S. T. Nguyen and J. T. Hupp, Chem. Soc. Rev., 2009, 38, 1450-1459.

14 Z. Kang, L. Fan and D. Sun, J. Mater. Chem. A, 2017, 5, 10073-10091.

15 Z. R. Herm, E. D. Bloch and J. R. Long, Chem. Mater., 2014, 26, 323-338.

16 R. Ricco, K. Konstas, M. J. Styles, J. J. Richardson, R. Babarao, K. Suzuki, P. Scopece and P. Falcaro, J. Mater. Chem. A, 2015, 3, 19822-19831.

17 H. C. Zhou, J. R. Long and O. M. Yaghi, Chem. Rev., 2012, 112, 673-674.
18 K. K. Tanabe and S. M. Cohen, Chem. Soc. Rev., 2011, 40, 498-519.

19 D. J. Tranchemontagne, J. L. Mendoza-Cortes, M. O'Keeffe and O. M. Yaghi, Chem. Soc. Rev., 2009, 38, 1257-1283.

20 Z. Q. Wang and S. M. Cohen, Chem. Soc. Rev., 2009, 38, 1315-1329.

21 H.-C. Lee, J. Hwang, U. Schilde, M. Antonietti, K. Matyjaszewski and B. V. K. J. Schmidt, Chem. Mater., 2018, 30, 2983-2994.

22 N. Ding, H. Li, X. Feng, Q. Wang, S. Wang, L. Ma, J. Zhou and B. Wang, J. Am. Chem. Soc., 2016, 138, 10100-10103.

23 T. Uemura, Y. Ono, K. Kitagawa and S. Kitagawa, Macromolecules, 2008, 41, 87-94.

24 T. Uemura, Y. Ono and S. Kitagawa, Chem. Lett., 2008, 37, 616-617.

25 T. Uemura, R. Kitaura, Y. Ohta, M. Nagaoka and S. Kitagawa, Angew. Chem., Int. Ed., 2006, 45, 4112-4116.

26 T. Kitao, M. W. A. MacLean, K. Nakata, M. Takayanagi, M. Nagaoka and T. Uemura, J. Am. Chem. Soc., 2020, 142, 5509-5514.

27 X. Zhang, T. Kitao, D. Piga, R. Hongu, S. Bracco, A. Comotti, P. Sozzani and T. Uemura, Chem. Sci., 2020, 11, 10844-10849.

28 T. Uemura, N. Yanai and S. Kitagawa, Chem. Soc. Rev., 2009, 38, 1228-1236.

29 T. Uemura, D. Hiramatsu, Y. Kubota, M. Takata and S. Kitagawa, Angew. Chem., Int. Ed., 2007, 46, 49874990.

30 T. Uemura, T. Kaseda and S. Kitagawa, Chem. Mater., 2013, 25, 3772-3776.

31 T. S. Wang, M. Farajollahi, S. Henke, T. T. Zhu, S. R. Bajpe, S. J. Sun, J. S. Barnard, J. S. Lee, J. D. W. Madden, A. K. Cheetham and S. K. Smoukov, Mater. Horiz., 2017, 4, 64-71.

32 C. J. Lu, T. Ben, S. X. Xu and S. L. Qiu, Angew. Chem., Int. Ed., 2014, 53, 6454-6458.

33 H. Gliemann and C. Woll, Mater. Today, 2012, 15, 110-116. 34 R. Haldar, B. Sen, S. Hurrle, T. Kitao, R. Sankhla, B. Kühl, A. Welle, S. Heissler, G. Brenner-Weiß, P. Thissen, T. Uemura, H. Gliemann, C. Barner-Kowollik and C. Wöll, Eur. Polym. J., 2018, 109, 162-168.

35 Z.-G. Gu, W.-Q. Fu, M. Liu and J. Zhang, Chem. Commun., 2017, 53, 1470-1473.

36 B. Liu, O. Shekhah, H. K. Arslan, J. X. Liu, C. Woll and R. A. Fischer, Angew. Chem., Int. Ed., 2012, 51, 807-810.

37 A. Betard and R. A. Fischer, Chem. Rev., 2012, 112, 10551083.

38 J. X. Liu and C. Woll, Chem. Soc. Rev., 2017, 46, 5730-5770.

39 O. Shekhah, J. Liu, R. A. Fischer and C. Woll, Chem. Soc. Rev., 2011, 40, 1081-1106.

40 B. Liu and R. A. Fischer, Sci. China: Chem., 2011, 54, 18511866.

41 K. Vanommeslaeghe and A. D. MacKerell Jr., J. Chem. Inf. Model., 2012, 52, 3144-3154.

42 K. Vanommeslaeghe, E. P. Raman and A. D. MacKerell Jr., J. Chem. Inf. Model., 2012, 52, 3155-3168. 
43 W. Yu, X. He, K. Vanommeslaeghe and A. D. MacKerell Jr., J. Comput. Chem., 2012, 33, 2451-2468.

44 I. Soteras Gutiérrez, F. Y. Lin, K. Vanommeslaeghe, J. A. Lemkul, K. A. Armacost, C. L. Brooks 3rd and A. D. MacKerell Jr., Bioorg. Med. Chem., 2016, 24, 48124825 .

45 Z. Wang, A. Błaszczyk, O. Fuhr, S. Heissler, C. Wöll and M. Mayor, Nat. Commun., 2017, 8, 14442.

46 S. Garg, H. Schwartz, M. Kozlowska, A. B. Kanj, K. Müller, W. Wenzel, U. Ruschewitz and L. Heinke, Angew. Chem., Int. Ed., 2019, 58, 1193-1197.

47 O. Shekhah, H. Wang, S. Kowarik, F. Schreiber, M. Paulus, M. Tolan, C. Sternemann, F. Evers, D. Zacher, R. A. Fischer and C. Wöll, J. Am. Chem. Soc., 2007, 129, 15118-15119.

48 M. Kind and C. Wöll, Prog. Surf. Sci., 2009, 84, 230-278.

49 H. K. Arslan, O. Shekhah, D. C. F. Wieland, M. Paulus, C. Sternemann, M. A. Schroer, S. Tiemeyer, M. Tolan, R. A. Fischer and C. Wöll, J. Am. Chem. Soc., 2011, 133, 8158-8161.

50 C. I. Simionescu, V. Bulacovschi, M. Grovu-ivanoiu and A. Stanciu, J. Macromol. Sci., Part A, 1987, 24, 611-622.
51 J. X. Liu, B. Lukose, O. Shekhah, H. K. Arslan, P. Weidler, H. Gliemann, S. Brase, S. Grosjean, A. Godt, X. L. Feng, K. Mullen, I. B. Magdau, T. Heine and C. Woll, Sci. Rep., 2012, 2, 5 .

52 M. Maes, S. Schouteden, K. Hirai, S. Furukawa, S. Kitagawa and D. E. De Vos, Langmuir, 2011, 27, 9083-9087.

53 S. D. Worrall, M. A. Bissett, P. I. Hill, A. P. Rooney, S. J. Haigh, M. P. Attfield and R. A. W. Dryfe, Electrochim. Acta, 2016, 222, 361-369.

54 J. Liu, T. Wächter, A. Irmler, P. G. Weidler, H. Gliemann, F. Pauly, V. Mugnaini, M. Zharnikov and C. Wöll, ACS Appl. Mater. Interfaces, 2015, 7, 9824-9830.

55 A. B. Kanj, R. Verma, M. Liu, J. Helfferich, W. Wenzel and L. Heinke, Nano Lett., 2019, 19, 2114-2120.

56 A. A. Talin, A. Centrone, A. C. Ford, M. E. Foster, V. Stavila, P. Haney, R. A. Kinney, V. Szalai, F. El Gabaly, H. P. Yoon, F. Léonard and M. D. Allendorf, Science, 2014, 343, 66-69.

57 A. Knebel, B. Geppert, K. Volgmann, D. I. Kolokolov, A. G. Stepanov, J. Twiefel, P. Heitjans, D. Volkmer and J. Caro, Science, 2017, 358, 347-351. 